\title{
How to Assign a (3+1)D Superspace Group to an Incommensurately Modulated Biological Macromolecular Crystal
}

Gloria E. O. Borgstahl, Eppley Institute for Research in Cancer, Omaha, NE Jason C. Porta, Purdue University, West Lafayette, IN

Jeffrey J. Lovelace, Eppley Institute for Research in Cancer, Omaha, NE

Periodic crystal diffraction is described using a three dimensional (3D) unit cell and 3D space group symmetry. Incommensurately modulated crystals are a subset of aperiodic crystals that need four to six dimensions to describe the observed diffraction pattern and they have characteristic satellite reflections that are offset from the main 3D lattice reflections. These satellites have a nonintegral relationship to the main lattice and require a q vector(s) for processing. Incommensurately modulated biological macromolecular crystals have been frequently observed but so far not solved. We have been spearheading an initiative to solve this type of crystal. The first step toward structure solution is to collect the diffraction data making sure that the satellite reflections are well separated from the main reflections. Once collected, the reflections can be indexed and integrated with Eval15 and then scaled with SADABS, at which point the assignment of the superspace group is needed. The most common form of modulation is in only one extra direction and can be described with a $(3+1) \mathrm{D}$ superspace group. The $(3+1) \mathrm{D}$ superspace groups for chemical crystallographers are fully described in Volume $\mathrm{C}$ of the International Tables for Crystallography in Chapter 9.8. This text includes all types of crystallographic symmetry elements found in small molecule crystals and can be difficult for structural biologists to understand and apply to their crystals. Here we provide an explanation for structural biologists that includes only the subset of biological symmetry elements and demonstrate the application to a real-life example of an incommensurately modulated protein crystal of Profilin:Actin. 